\title{
Acoustic characteristics of Italian Parkinsonian speech: a study on early-stage patients
}

\author{
Marta Maffia ${ }^{1}$, Rosa De $\mathrm{Micco}^{2}$, Alessandro Tessitore ${ }^{2}$, Anna De Meo \\ ${ }^{1}$ Department of Literary, Linguistics and Comparative Studies, University of Naples \\ "L'Orientale", Italy \\ ${ }^{2}$ Department of Advanced Medical and Surgical Sciences, University of Campania \\ "Luigi Vanvitelli", Italy \\ https://doi.org/10.36505/ExLing-2020/11/0029/000444
}

\begin{abstract}
Parkinson's Disease (PD) is a neurological illness which also has effects on speech production, resulting in segmental and suprasegmental abnormalities. The aim of the current study is to test the validity of two acoustic parameters $\% \mathrm{~V}$ and $\mathrm{VtoV}$ - for the detection of rhythmical variation in early-stage PD speech, in comparison to healthy speech. 40 Italian native speakers were enrolled in the research, 20 early-stage PD subjects and 20 neurologically healthy and matched controls, and a corpus of read speech was collected. The results of voice analysis confirmed an alteration of vocalic duration and $\% \mathrm{~V}$ in $\mathrm{PD}$ productions. In particular, $\% \mathrm{~V}$ could be a reliable cue for PD speech characterization, even at the very early onset of the disease.
\end{abstract}

Keywords: Parkinson's disease, language pathology, acoustic analysis, speech rhythm, early diagnosis

\section{Introduction}

Parkinson's Disease (PD) is recognized as the second most common neurodegenerative disorder after Alzheimer's Disease, with a point prevalence ranging from $0.25-4 \%$ between age of 65-80 (de Lau \& Breteler 2006).

Degeneration of nigrostriatal dopaminergic neurons, which results in disruption of basal ganglia-thalamo-cortical loops, underlies the classical motor signs and symptoms of PD (i.e. bradykinesia, rigidity, tremor and postural instability). As reported in experimental studies on different languages, PDrelated dysarthria also has effects on the three major anatomic subsystems governing speech motor control: respiration, phonation and articulation. At both the segmental and the suprasegmental level of speech, the hypokinesia and the resulting decreased amplitude of motion of lips, tongue and jaw may cause a variety of deficits, such as imprecise vowel and consonant articulation, reduced or abnormal pitch variability, speech fluency and speech rate (see, among the others, Skodda et al. 2012, Liss et al. 2009).

ExLing 2020: Proceedings of $11^{\text {th }}$ International Conference of Experimental Linguistics, 12-14 October 2020, Athens, Greece 
In this framework, it has been suggested that the observation of some acoustic parameters' variation may potentially provide a sustainable and noninvasive diagnostic tool, in support of clinical assessment, even in the very early stages of the disease, when the neurodegeneration is yet started and spread throughout the nervous system but still there are no motor signs (Harel et al. 2004).

Previous researches on different languages and on mild-to-severe PD patients have shown the effectiveness of two acoustic parameters, vowel percentage $(\% \mathrm{~V})$ and the mean interval between two consecutive vowel onset points (VtoV), in the description of rhythmic variations of PD speech, compared to healthy speech (Pettorino et al. 2016, Pettorino et al. 2017).

The purpose of the present study is to verify the validity of the two acoustic parameters, $\% \mathrm{~V}$ and $\mathrm{VtoV}$, even for the speech characterization of Italian subjects with early-stage PD.

\section{Methods}

\section{Subjects}

The data for the present research were collected from 40 speakers: 20 individuals diagnosed with PD and 20 age and sex-matched healthy controls (HC) with no history of neurological or speech disorders. All subjects were Italian native speakers residing in Campania region and they all gave written consent to the data collection procedure.

Patients were recruited from an ongoing longitudinal study enrolling nondemented early PD patients diagnosed according to the modified diagnostic criteria of the UK Parkinson's Disease Society Brain Bank (Gibb \& Lees, 1988) at the Movement Disorders Unit of the First Division of Neurology at the University of Campania "Luigi Vanvitelli" (Naples, Italy). They underwent an extensive motor and non-motor assessment with validated PD-related scales.

\section{Speech data collection and analysis}

Each subject was instructed to read aloud from a printed sheet an expository text (about 350 syllables), chosen for having a low level of syntactic and lexical complexity and a very simple content. They were encouraged to speak in their normal, conversational voice, as spontaneously as possible and at comfortable loudness. The speech samples were recorded on a standard personal computer in a quiet room of University "Luigi Vanvitelli", by means of the software Praat (Boersma \& Weenink 2009) at a $44100 \mathrm{~Hz}$ sampling rate. Sociolinguistic information on each speaker were also obtained with a questionnaire.

Patients performed speech assessment while taking their regular dopaminergic medications.

All the read speech samples were manually labelled to identify consonantal and vocalic intervals, through the visual inspection of speech spectrograms and waveforms. Approximants were completely avoided in the chosen text, while diphthongs were considered as a single vocalic interval. In $\mathrm{V}+$ nasal consonant 
sequences, the nasalized portion of the vowel was assigned to the $\mathrm{V}$ interval. As for initial voiced stop consonants, the first boundary was considered to be the onset of the glottal pulses. Post-pausal voiceless plosives were assigned a duration equal to the mean value of single plosives in the same utterance.

Once extracted the durations of all consonantal and vocalic portions, $\% \mathrm{~V}$ and VtoV were obtained using a Praat script. Disfluences and silent pauses were not considered for the calculation of these two parameters.

\section{Statistical analysis of clinical and speech data}

T-test or $\mathrm{X}^{2}$ were computed to compare clinical and speech data as appropriate. A $p<0.05$ was considered as statistically significant. Analyses were performed with SPSS version 23.

\section{Results}

The results of acoustic analysis are reported in Table 1, along with demographic and clinical data for all participants.

Table 1. Demographic, clinical and acoustic features of HC and PD patients.

\begin{tabular}{llccc}
\hline & & $\begin{array}{c}\text { HC }(\mathrm{n}=20) \\
\text { mean } \pm \text { SD }\end{array}$ & $\begin{array}{c}\text { PD }(\mathrm{n}=20) \\
\text { mean } \pm \text { SD }\end{array}$ & p-value \\
\hline Demographic data & Age & $64.8 \pm 5.9$ & $63.8 \pm 10.9$ & 0.713 \\
& Sex (M/F) & $8 / 12$ & $12 / 8$ & 0.205 \\
& Disease duration & - & $31.7 \pm 18.9$ & - \\
(months) & & $1.9 \pm 0.5$ & - \\
Clinical data & H\&Y stage & - & $25.0 \pm 8.2$ & - \\
& UPDRS III & - & $24.4 \pm 4.3$ & - \\
& MoCA total & - & $5.2 \pm 6.2$ & - \\
Acoustic data & BDI & - & $289.9 \pm 152.3$ & - \\
& LEDD total & - & $78.3 \pm 129.1$ & - \\
& LEDD DA & - & $91.3 \pm 12.1$ & $\mathbf{0 . 0 0 4}$ \\
& durV (ms) & $81.6 \pm 7.9$ & $93.5 \pm 12.7$ & $\mathbf{0 . 0 0 7}$ \\
& durC (ms) & $103.2 \pm 9.1$ & $49.8 \pm 1.7$ & $<\mathbf{0 . 0 0 1}$ \\
& $\%$ VV & $44.7 \pm 1.3$ & 49.7 & 0.889 \\
\hline
\end{tabular}

H\&Y: Hoehn \& Yahr; UPDRS: Unified Parkinson's Disease Rating Scale; MoCA: Montreal Cognitive Assessment; BDI: Beck depression inventory; LEDD: Levodopa Equivalent Daily Dose; DA: dopamine-agonist. Significant differences are reported in bold.

The $\% \mathrm{~V}$ is, thus, confirmed to be the parameter that changes most significantly in the two groups of speakers, being distinctly higher in PD speech (from $47.8 \%$ to $54.2 \%$ ) than in HC productions (from $42.3 \%$ to $47.2 \%$ ). Despite a high variability across speakers within both groups, on average, consonants are shortened in PD speech, as a possible consequence of consonant weakening, while vowels are significantly longer in PD productions than in $\mathrm{HC}$ speech. 
With regard to VtoV, the two groups of participants present very similar mean values and the same speech rate variability.

\section{Discussion and conclusions}

First results suggest that $\% \mathrm{~V}$ is specifically altered in PD patients even at the disease onset. Dopaminergic pathways, which are known to be primarily affected at PD onset, may be also crucially involved in speech processes, altering very early the articulatory passage from the static phase, represented by vowels, to the dynamic phase of consonant production. This would account for the rhythmic characterization of PD speech, in which more time is spent on vocalic sounds with respect to healthy controls. Further investigations on larger PD samples are needed to support our observations.

\section{Acknowledgements}

We would like to thank Prof. Gioacchino Tedeschi and Prof. Massimo Pettorino for their precious support.

\section{References}

Boersma, P., Weenink, D. 2020. Praat: doing phonetics by computer [Computer program]. Version 6.1.20beta, retrieved 10 September 2020 from http://www.praat.org/.

de Lau, L. M., Breteler, M.M. 2006. Epidemiology of Parkinson's disease. Lancet Neurol 5, 525-535.

Gibb, W.R., Lees, A.J. 1988. A comparison of clinical and pathological features of young- and old-onset Parkinson's disease. Neurology 38, 1402-1406.

Harel, B.T., Cannizzaro, M.S., Cohen, H., Reilly, N., Snyder, P.J. 2004. Acoustic characteristics of Parkinsonian speech: a potential biomarker of early disease progression and treatment. Journal of Neurolinguistics 17, 439-453.

Liss, J.M., White, L., Mattys, S.L., Lansford, K., Lotto, A.J., Spitzer, S.M., Caviness, J.N. 2009. Quantifying speech rhythm abnormalities in the dysarthrias. Journal of Speech, Language, and Hearing Research 52, 1334-1352.

Pettorino, M., Busà, M.G., Pellegrino, E. 2016. Speech Rhythm in Parkinson's Disease: A Study on Italian. Proc. of the $17^{\text {th }}$ Annual Conference of the International Speech Communication Association, San Francisco, USA, 1958-1961.

Pettorino, M., Gu, W., Półrola, P., Fan, P. 2017. Rhythmic Characteristics of Parkinsonian Speech: A Study on Mandarin and Polish. Proc. of the $18^{\text {th }}$ Annual Conference of the International Speech Communication Association, Stockholm, Sweden, 3172-3176.

Skodda, S., Gronheit, W., Schlegel, U. 2012. Impairment of vowel articulation as a possible marker of disease progression in Parkinson's Disease, PLoS ONE 7/2,1-8. 